\title{
Integrated Analysis of a circRNA-miRNA-mRNA Axis in Intervertebral Disc Degeneration
}

\section{Bizhi Tu} \\ Anhui Medical University

\section{Fei Gao} \\ Anhui Medical University \\ Jun Qian \\ Anhui Medical University
}

Laifu Wei ( $\square$ 1213059252@163.com )

Anhui Medical University https://orcid.org/0000-0003-0218-5726

\section{Research article}

Keywords: intervertebral disc degeneration, circRNA, GEO

Posted Date: December 18th, 2020

DOl: https://doi.org/10.21203/rs.3.rs-129853/v1

License: (9) This work is licensed under a Creative Commons Attribution 4.0 International License. Read Full License 


\section{Abstract}

Background: Low back pain (LBP) is a common symptom in daily life and one of the primary causes is intervertebral disc degeneration (IDD). Growing studies have indicated that circular RNAs (circRNAs) are intimately associated with IDD; however, the underlying mechanism has not yet been elucidated. We aimed to explore how circRNAs regulate IDD in an effort to provide novel insight for clinical diagnosis and treatment.

Methods: The sequencing data of circRNAs, microRNAs (miRNAs), and mRNA were acquired from Gene Expression Omnibus (GEO) datasets. By analyzing the dataset consisting of a control group and degenerated group, differentially expressed circRNAs, miRNAs, and mRNAs were collected, and then the intersection of circRNAs, miRNAs, and mRNAs was screened. According to these intersectional RNAs, we constructed an integrally circRNA-miRNA-mRNA network. Finally, using Gene Ontology (GO) and Kyoto Encyclopedia of Genes and Genomes (KEGG) analyses, we further clarified functions of the intersectional mRNA in IDD.

Results: we obtained 620 differentially expressed circRNAs(DEcircRNAs), 13 miRNA (DEmiRNA), 273 mRNAs(DEmRNAs), 12 intersectional miRNAs, and 47 intersectional mRNAs. Finally, based on interactional 8 circRNA, 5 miRNAs and 15 mRNAs, an integrally circRNA-miRNA-mRNA network was constructed. Eight circRNAs, contained hsa_circ_0032254, hsa_circ_0003183, hsa_circ_0032253, hsa_circ_0001293, hsa_circ_0004565, hsa_circ_0091570, hsa_circ_0077526, and hsa_circ_0057552, may regulate IDD onset and progression by acting as competing endogenous RNAs. The results of GO and KEGG analyses implied that the targeted genes might significantly correlate to IDD.

Conclusion: our findings improved a better understanding of the circRNA-related ceRNA regulatory mechanism in IDD and offered possible targets for IDD treatment.

\section{Introduction}

Low back pain is the primary cause of disability in developed countries and a growing number of people are affected worldwide [1]. Although the reason for low back pain remains unclear, some research has demonstrated that low back pain is correlated with intervertebral disc degeneration (IDD) [2-7]. Anatomically, the intervertebral disc consists of three components: I) an inner cord termed the nucleus pulposus (NP), II) annulus fibrous surrounding the NP, and III) the cartilaginous endplate [8,9]. Nucleus pulposus cells (NPCs) in the NP generate several components of the extracellular matrix (ECM) including collagen 2 and proteoglycans in physiological conditions $[9,10]$. These proteins are vital in maintaining disc structure and resistance to various mechanical compression forms from external factors [11]. However, multiple abnormal stimuli, such as neutrophil proteases, can upregulate the expression of inflammatory cytokines (e.g., IL-1 $\beta$, TNF-a), and then breakdown the balance of NPCs through several factors including matrix metalloproteinases (MMPs), collagen II, metalloproteinases with thrombospondin motifs (ADAMTS), and aggrecan. These imbalances trigger or accelerate the 
degeneration of the intervertebral disc $[12,13]$. However, the deeper pathological mechanism underlying IDD is still unknown. Thus, it is essential to seek a more effective way to moderate the inflammatory response and reverse the NP microenvironment imbalance.

Circular RNAs (circRNAs) are a type of single-stranded loop RNA that are primarily considered to act as competing endogenous RNAs (ceRNAs), binding microRNAs (miRNAs) to further regulate the expressed level of targeted mRNAs [14-17]. For instance, upregulation of circ-4099 has been shown to inhibit IDD development by modulating the circ-4099-miR-616-5p-Sox9 network [18]. An increasing number of reviews demonstrated that circRNAs have a crucial role in the pathogenesis of IDD $[19,20]$. In order to better know the underlying mechanism of circRNAs in IDD, we generated a circRNA-miRNA-mRNA network by sequencing data from the GEO database. This study may provide a new target in the pathogenesis and therapeutic strategies of IDD.

\section{Methods}

\section{Data acquisition}

Three expression profiles of circRNAs (GSE67566), miRNAs (GSE63492), and mRNAs (GSE124272) were downloaded from the GEO database (http://www.ncbi.nlm.nih.gov/geo/). And then we analyzed these data by limma R package. Gene symbols corresponding to the probes were obtained using the annotation information in the platform.

GSE67566 (platform: GPL19978) and GSE63492 (platform: GPL19449) consisted each of five pairs of control and degenerated NP samples. GSE124272 (platform: GPL21185) consisted of eight normal and degenerated NP tissues.

\section{Identification of the differential expression of circRNAs, miRNAs and mRNAs}

The differentially expressed profiles of circRNAs, miRNAs, and mRNAs between normal and degenerated NP tissues were acquired using the limma $R$ package. It is necessary and statistically significant thresholds that Log fold change $(\mathrm{FC})>1.0$ and adjusted $\mathrm{P}$-value $<0.05$ were needed.

\section{Construction of the ceRNA network}

The circBase website (http://www.circbase.org/) was used to obtain information regarding differentially expressed circRNAs(DEcircRNAs), including the chromosomal position of each DEcircRNA and precise localization of DEcircRNAs at the gene sequence level. Then, we determined the miRNAs (MREs) that combined with DEcircRNAs by the cancer-specific circRNA database (CSCD)

(https://gb.whu.edu.cn/CSCD/). We first overlapped MREs and differentially expressed miRNAs (DEmiRNAs) for potential targeted miRNAs; then, the potential target mRNA was obtained using the TargetScan and miRDB databases. At last, the targeted genes were identified by the interaction of potential target mRNAs and DEmRNAs. Therefore, the regulated circRNA-miRNA-mRNA network was 
constructed by screening out intersectional circRNAs, miRNAs, and mRNAs. The regulatory network is presented using Cytoscape 3.7.1.

\section{Functional and pathway enrichment analyses form databases of GO and KEGG}

To ascertain the function of targeted genes in intervertebral disc degeneration, Kyoto Encyclopedia of Genes and Genomes (KEGG) pathway and Gene Ontology (GO) analyses were performed using the online tool Database of DAVID (http://david.abcc.ncifcrf.gov, version 6.8). An adjusted P-value $<0.05$ was regarded as statistically significant.

\section{Results}

\section{Differential expression analysis}

Table 1 listed the expressional basic information of the circRNAs, miRNAs and mRNAs in normal and degenerated NP tissues, which come from three sequencing data. Based on Log [FC] >1.0 and adjusted Pvalue $<0.05$, we identified 620 DEcircRNAs including 337 upregulated and 283 downregulated DEcircRNAs (Fig. 1). The basic information of the 620 DEcircRNAs was downloaded from circBase (http://www.circbase.org/cgi-bin/listsearch.cgi). Using the CSCD database, we found a total of 2,390 miRNAs combined with DEcircRNAs. In the differentially expressed dataset of miRNAs, eight DEmiRNAs were upregulated and five DEmiRNAs were downregulated (Fig. 2). Twelve overlapped miRNAs were collected using the limma R package (Fig. 3). Using the TargetScan and miRDB databases, a total of 5,203 targeted miRNAs were predicted. We find 182 mRNAs upregulated and 91 mRNAs downregulated in the IDD groups (Fig. 4). Finally, 47 intersected genes were identified (Fig. 5).

Table 1

Basic information of the 3 microarray datasets from GEO

\begin{tabular}{|llll|}
\hline Data source & Platform & Series & Sample size $(\mathrm{T} / \mathrm{N})$ \\
\hline CircRNA & GPL19978 & GSE67566 & $5 / 5$ \\
miRNA & GPL19449 & GSE63492 & $5 / 5$ \\
mRNA & GPL21185 & GSE124272 & $8 / 8$ \\
\hline
\end{tabular}

\section{Construction of the ceRNA network}

To better understand the effect of circRNAs on IDD, we constructed an integrated circRNA-miRNA-mRNA network by linking circRNA-miRNA and miRNA-mRNA. The network contained eight circRNAs (two upregulated and six downregulated), five miRNAs (two upregulated and three downregulated), and 15 mRNAs (eight upregulated and seven downregulated), which are shown using Cytoscape 3.7.1 (Fig. 6). 


\section{Functional and pathway enrichment analysis}

The mainly enriched genes based on $\mathrm{GO}$ analysis are shown. The first five gathered terms were male sex determination, positive regulation of myelination, sex determination, regulation of myelination, positive regulation of nervous system process in the biological process (BP) category (Fig. 7a); specific granule, tertiary granule, host cell cytoplasm part, host cell cytoplasm, and host intracellular part in the cellular component (CC) category (Fig. 7b); and nuclear receptor activity, ligand-activated transcription factor activity, steroid hormone receptor activity, RAGE receptor binding, and S100 protein binding in the molecular function (MF) category (Fig. 7c). The p53 signaling pathway and cortisol synthesis and secretion were strongly enriched in KEGG pathway analysis (Fig. 7d).

\section{Discussion}

The circRNAs were originally discovered in plant viroid and yeast mitochondrial RNAs $[7,21]$ and have been rarely tested by transcriptomic polyadenylated RNA profiling owing to the lack of polyadenylation at their 3 ' ends [22-25]. With the development of novel technology including calculation procedure and high-throughput sequencing [22], non-polyadenylated human RNA sequencing analysis and the signals of RNA sequencing can be obtained to assess back-spliced exons [26] or stabilized introns [27]. With their covalently closed structure, circRNAs are highly stable non-coding RNAs that play a critical role in cell and tissue development and several disease processes, including cancer, IDD, and neurodegeneration [19, 2830].

Although the molecular mechanisms of circRNAs in mammalian cells remain mostly unclear, circRNA regulation of the level of mRNAs by a circRNA-miRNA-mRNA network may be an important mechanism in the initiation and development of IDD. Wang et al. showed that the circular RNA SEMA4B could weaken the impact of IL-1 $\beta$ on NPC senescence, ECM and aggrecan degradation in IDD via inhibition of miR-431 (a targeted miRNA of circRNA SEMA4B) [20]. Moreover, circRNA_104670 suppressed the expression level of collagen II and the proliferation of NPCs, and promoted NPCs' apoptosis by circRNA_104670/miR-173p/MMP-2[19]. The circRNA VMA21, regarded as a sponge of mir-200c, plays a role in regulating the level of inflammatory cytokines by targeting mir200c and XIAP [31]. To research the role of circRNAs in IDD, we identified 620 DEcircRNAs by comparing normal and degenerated groups' expression profiles. Similarly, we discovered 13 DEmiRNAs and 273 DEmRNAs. To further understand the underlying mechanisms of these RNA molecules, we constructed a circRNA-miRNA-mRNA network for IDD. Our research provided several novel targets and molecular biomarkers for IDD. The hsa_circ_0091570, a sponge of miR-1307 that acted to regulate ISM1 expression in hepatocellular cancer, was screened from the ceRNA network constructed by 8 circRNAs, 13 miRNAs and 15 genes [32]. Three additional genes, CHEK1, TSGA10, and GSTCD, have been reported to downregulate genes that inhibit cellular proliferation or reduce cell numbers [33-35]. what's more, NPCs' proliferation and apoptosis play a key role in IDD [36]. Meanwhile, we confirmed that the level of these genes was downregulated in IDD. Thus, Hsa_circ_0091570 may suppress NPC proliferation by downregulating the expression of CHEK1, TSGA10, and GSTCD. The regulated network of hsa_circ_0091570-hsa-miR-508-5p-CHEK1/TSGA10/GSTCD was constructed and 
the RNAs (circRNAs and miRNAs) and genes in the network may all be novel targets for IDD. In parallel, Li et al. reported that miR-183-3p regulates autophagy in gastric cancer cells by the PI3K/AKT/mTOR pathway [37]. According to previous research, the PI3K/AKT/mTOR pathway has a significant effect on cell growth, survival, metabolism, proliferation, and angiogenesis [38]. Moreover, overexpressed PLG can promote the proliferation of keratinocytes [39]. We constructed a network with downregulated hsa_circ_0004565, upregulated hsa-miR-183-3p, and downregulated PLG. However, whether hsa_circ_0004565 depresses the proliferation of NPCs via downregulating the expression of PLG which further regulates $\mathrm{PI3K} / \mathrm{AKT} / \mathrm{mTOR}$ signaling is unclear yet. Some studies have shown that S100B regulates inflammation by increasing the expression of TNF- $\alpha$ and IL-1 $\beta$ during osteoarthritis [40]. Overexpression of TNF- $\alpha$ and IL-1 $\beta$ is also significant in the breaking of intervertebral discs [13]. However, we found that the level of S100B expression was decreased in IDD. The S100B-related ceRNA network (hsa_circ_0003183/hsa_circ_0032253/hsa_circ_0001293-hsa-miR-4534-S100B) was constructed. We discover that circRNA (hsa_circ_0003183/hsa_circ_0032253/hsa_circ_0001293) may act as an independent protective factor to inhibit IDD by decreasing the expression of S100B. Many miRNAs are involved in different diseases, such as hsa-miR-1827 and hsa-miR-508-5p[41, 42]. In our research, we found that hsa-miR-1827 regulated the seven upregulated target genes, including GBP6, CEACAM8, C7orf34, KCNV2, OLFM4, NR5A1, and ZNF488. Studies have demonstrated that hsa-miR-1827 and its target(s) are closely associated with breast cancer, cervical cancer, and tongue squamous cell carcinoma [42-48]; we also explored whether these genes or miRNAs are involved in IDD, and then we further verify the connection of circRNA and IDD by constructing an integrated circRNA-miRNA-mRNA network. For example, the high expression of target genes CEACAM8 and OLFM4 plays a crucial role in the inflammatory process by increasing the level of inflammatory cytokines [49,50]. A great number of inflammatory cytokines promote extracellular matrix degradation and disorder of NPCs' inner or outer environment, which plays a significant role in IDD [51]. We also constructed the hsa_circ_0057552-hsamiR-1827-GBP6/CEACAM8/C7orf34/KCNV2/OLFM4/NR5A1/ZNF488 network. We find that hsa_circ_0057552 may take part in the inflammatory process and degeneration in IDD by enhancing the expression of CEACAM8 and OLFM4. Additionally, it was reported that ZNF488-overexpressing cells could increase the expression of collagen IV [52] which obviously decreased in IDD [53]. Hence, hsa_circ_0057552 may be a protective factor to resist IDD by increasing the expression of ZNF488. Although different miRNAs or genes involved in the network have been reported in different studies, this ceRNA-regulated network has not yet been reported in IDD. Moreover, the molecular circadian function of the hsa_circ_0057552-hsa-miR-1827-GBP6/CEACAM8/C7orf34/KCNV2/OLFM4/NR5A1/ZNF488 network remains unclear and will require further research.

KEGG analysis showed that the majority of genes were enriched in the p53 signaling pathway. Although p53 is a nuclear transcription factor that plays a vital role in regulating cell cycle progression, senescence, and cell death [54], its role in IDD will require further study. GO analysis revealed genes mostly focused on the regulation of the nervous system process, myelination, and steroid hormone receptor activity.

In conclusion, this research identified several circRNA-miRNA-mRNA interaction axes, such as hsa_circ_0091570-hsa-miR-508-5p-CHEK1, hsa_circ_0057552-hsa-miR-1827-GBP6, and 
hsa_circ_0003183-hsa-miR-4534-S100B, which provided novel insight into the pathological mechanism and clinical treatment of IDD.

\section{Limitation}

This study has some restricted conditions. At first, the number of samples is not enough. We need an additional cohort to further validate the expressed level of circRNAs, miRNAs and mRNAs in IDD. Secondly, based on our preliminary screening study, the interactions of identified ceRNA should be confirmed in future studies.

\section{Abbreviations}

LBP: Low back pain; IDD: Intervertebral disc degeneration; GEO: Gene Expression Omnibus; KEGG: Kyoto Encyclopedia of Genes and Genomes; GO: Gene Ontology; BP: Biological process; CC: Cellular component; MF: Molecular function;

\section{Declarations}

\section{Acknowledgments}

We thank Department of Spine Surgery, The First Affiliated Hospital of Anhui Medical University for its grateful supports for the project.

\section{Funding}

This project was supported by the National Natural Science Foundation of China (grant numbers 81471273 and 81671204$)$.

\section{Availability of data and materials}

All datasets used and/or analyzed during the present study are available from the corresponding author on reasonable request.

\section{Authors' contributions}

JQ conceived the study idea. LF collected the data. LF, BZ, and FG contributed to the analysis of the data as well as wrote the initial draft with all authors providing critical feedback and edits to subsequent revisions. All authors approved the final draft of the manuscript. All authors are accountable for all aspects of the work in ensuring related questions accuracy or integrity. Any parts of the work are appropriately investigated and resolved. JQ is the guarantor. The corresponding author attests that all listed authors meet authorship criteria and that no others meeting the criteria have been omitted.

\section{Ethics approval and consent to participate}


The date of this study is from online public datasets, which don't involve in ethics.

\section{Consent for publication}

Not applicable

\section{Competing interests}

The authors declare that they have no competing interests

\section{Publisher's Note}

Springer Nature remains neutral with regard to jurisdictional claims in published maps and institutional affiliations.

\section{Author details}

${ }^{1}$ Department of Orthopedics, The First Affiliated Hospital of Anhui Medical University, 81Meishan Road, Hefei 230022, Anhui, China. ${ }^{1 *}$ Department of Orthopedics, The First Affiliated Hospital of Anhui Medical University, 218Jixi Road, Hefei 230022, Anhui, China. Email: gjpaper@sina.cn

Tel. +8613955116514, Fax: 8613955116514

\section{References}

1. Hoy D, March L, Brooks P, Blyth F, Woolf A, Bain C, Williams G, Smith E, Vos T, Barendregt J et al: The global burden of low back pain: estimates from the Global Burden of Disease 2010 study. Ann Rheum Dis 2014, 73(6):968-974.

2. Wang Y, Videman T, Battie MC: ISSLS prize winner: Lumbar vertebral endplate lesions: associations with disc degeneration and back pain history. Spine (Phila Pa 1976) 2012, 37(17):1490-1496.

3. Scheele J, de Schepper El, van Meurs JB, Hofman A, Koes BW, Luijsterburg PA, Bierma-Zeinstra SM: Association between spinal morning stiffness and lumbar disc degeneration: the Rotterdam Study. Osteoarthritis Cartilage 2012, 20(9):982-987.

4. Livshits G, Popham M, Malkin I, Sambrook PN, Macgregor AJ, Spector T, Williams FM: Lumbar disc degeneration and genetic factors are the main risk factors for low back pain in women: the UK Twin Spine Study. Ann Rheum Dis 2011, 70(10):1740-1745.

5. Teraguchi M, Yoshimura N, Hashizume H, Muraki S, Yamada H, Minamide A, Oka H, Ishimoto Y, Nagata K, Kagotani R et al: Prevalence and distribution of intervertebral disc degeneration over the entire spine in a population-based cohort: the Wakayama Spine Study. Osteoarthritis Cartilage 2014, 22(1):104-110.

6. Luoma K, Riihimaki H, Luukkonen R, Raininko R, Viikari-Juntura E, Lamminen A: Low back pain in relation to lumbar disc degeneration. Spine (Phila Pa 1976) 2000, 25(4):487-492. 
7. Sanger HL, Klotz G, Riesner D, Gross HJ, Kleinschmidt AK: Viroids are single-stranded covalently closed circular RNA molecules existing as highly base-paired rod-like structures. Proceedings of the National Academy of Sciences of the United States of America 1976, 73(11):3852-3856.

8. Colombini A, Lombardi G, Corsi MM, Banfi G: Pathophysiology of the human intervertebral disc. Int J Biochem Cell Biol 2008, 40(5):837-842.

9. Boxberger Jl, Orlansky AS, Sen S, Elliott DM: Reduced nucleus pulposus glycosaminoglycan content alters intervertebral disc dynamic viscoelastic mechanics. Journal of Biomechanics 2009, 42(12):1941-1946.

10. Ding F, Shao ZW, Xiong LM: Cell death in intervertebral disc degeneration. Apoptosis 2013, 18(7):777785.

11. Hayes AJ, Benjamin M, Ralphs JR: Extracellular matrix in development of the intervertebral disc. Matrix Biol 2001, 20(2):107-121.

12. Dudek M, Yang N, Ruckshanthi JP, Williams J, Borysiewicz E, Wang P, Adamson A, Li J, Bateman JF, White MR et al: The intervertebral disc contains intrinsic circadian clocks that are regulated by age and cytokines and linked to degeneration. Ann Rheum Dis 2017, 76(3):576-584.

13. Johnson ZI, Schoepflin ZR, Choi H, Shapiro IM, Risbud MV: Disc in flames: Roles of TNF-alpha and IL-1 beta in intervertebral disc degeneration. Eur Cell Mater 2015, 30:104-116; discussion 116-107.

14. Hansen TB, Jensen TI, Clausen BH, Bramsen JB, Finsen B, Damgaard CK, Kjems J: Natural RNA circles function as efficient microRNA sponges. Nature 2013, 495(7441):384-388.

15. Memczak S, Jens M, Elefsinioti A, Torti F, Krueger J, Rybak A, Maier L, Mackowiak SD, Gregersen LH, Munschauer $\mathrm{M}$ et al: Circular RNAs are a large class of animal RNAs with regulatory potency. Nature 2013, 495(7441):333-338.

16. Zheng Q, Bao C, Guo W, Li S, Chen J, Chen B, Luo Y, Lyu D, Li Y, Shi G et al: Circular RNA profiling reveals an abundant circHIPK3 that regulates cell growth by sponging multiple miRNAs. Nat Commun 2016, 7(1).

17. Piwecka M, Glazar P, Hernandez-Miranda LR, Memczak S, Wolf SA, Rybak-Wolf A, Filipchyk A, Klironomos F, Cerda Jara CA, Fenske $P$ et al: Loss of a mammalian circular RNA locus causes miRNA deregulation and affects brain function. Science 2017, 357(6357).

18. Wang H, He P, Pan H, Long J, Wang J, Li Z, Liu H, Jiang W, Zheng Z: Circular RNA circ-4099 is induced by TNF-alpha and regulates ECM synthesis by blocking miR-616-5p inhibition of Sox9 in intervertebral disc degeneration. Exp Mol Med 2018, 50(4):27.

19. Song J, Wang H-L, Song K-H, Ding Z-W, Wang H-L, Ma X-S, Lu F-Z, Xia X-L, Wang Y-W, Fei Z et al: CircularRNA_104670 plays a critical role in intervertebral disc degeneration by functioning as a ceRNA. Experimental \& Molecular Medicine 2018, 50(8).

20. Wang X, Wang B, Zou M, Li J, Lu G, Zhang Q, Liu F, Lu C: CircSEMA4B targets miR-431 modulating IL1 beta-induced degradative changes in nucleus pulposus cells in intervertebral disc degeneration via Wnt pathway. Biochim Biophys Acta Mol Basis Dis 2018, 1864(11):3754-3768. 
21. Arnberg AC, Van Ommen GJ, Grivell LA, Van Bruggen EF, Borst P: Some yeast mitochondrial RNAs are circular. Cell 1980, 19(2):313-319.

22. Zhang XO, Wang HB, Zhang Y, Lu X, Chen LL, Yang L: Complementary sequence-mediated exon circularization. Cell 2014, 159(1):134-147.

23. Jeck WR, Sharpless NE: Detecting and characterizing circular RNAs. Nat Biotechnol 2014, 32(5):453461.

24. Wilhelm BT, Marguerat S, Watt S, Schubert F, Wood V, Goodhead I, Penkett CJ, Rogers J, Bahler J: Dynamic repertoire of a eukaryotic transcriptome surveyed at single-nucleotide resolution. Nature 2008, 453(7199):1239-1243.

25. Svensson V, Natarajan KN, Ly LH, Miragaia RJ, Labalette C, Macaulay IC, Cvejic A, Teichmann SA: Power analysis of single-cell RNA-sequencing experiments. Nat Methods 2017, 14(4):381-387.

26. Salzman J, Gawad C, Wang PL, Lacayo N, Brown PO: Circular RNAs are the predominant transcript isoform from hundreds of human genes in diverse cell types. PLoS One 2012, 7(2):e30733.

27. Zhang Y, Zhang XO, Chen T, Xiang JF, Yin QF, Xing YH, Zhu S, Yang L, Chen LL: Circular intronic long noncoding RNAs. Mol Cell 2013, 51(6):792-806.

28. Lukiw WJ: Circular RNA (circRNA) in Alzheimer's disease (AD). Front Genet 2013, 4:307.

29. Caiment F, Gaj S, Claessen S, Kleinjans J: High-throughput data integration of RNA-miRNA-circRNA reveals novel insights into mechanisms of benzo[a]pyrene-induced carcinogenicity. Nucleic Acids Res 2015, 43(5):2525-2534.

30. Barrett SP, Salzman J: Circular RNAs: analysis, expression and potential functions. Development 2016, 143(11):1838-1847.

31. Cheng X, Zhang L, Zhang K, Zhang G, Hu Y, Sun X, Zhao C, Li H, Li YM, Zhao J: Circular RNA VMA21 protects against intervertebral disc degeneration through targeting miR-200c and $X$ linked inhibitorof-apoptosis protein. Ann Rheum Dis 2018, 77(5):770-779.

32. Wang YG, Wang T, Ding M, Xiang SH, Shi M, Zhai B: hsa_circ_0091570 acts as a ceRNA to suppress hepatocellular cancer progression by sponging hsa-miR-1307. Cancer Lett 2019, 460:128-138.

33. Henry AP, Probert K, Stewart CE, Thakker D, Bhaker S, Azimi S, Hall IP, Sayers I: Defining a role for lung function associated gene GSTCD in cell homeostasis. Respir Res 2019, 20(1):172.

34. Wu D, Lin J, Zhu Y, Zhang H, Zhong Y: Expression of Testis-Specific Gene Antigen 10 (TSGA10) is Associated with Apoptosis and Cell Migration in Bladder Cancer Cells and Tumor Stage and Overall Survival in Patients with Bladder Cancer. Med Sci Monit 2019, 25:5289-5298.

35. Bai X, Wang J, Huo L, Xie Y, Xie W, Xu G, Wang M: Serine/Threonine Kinase CHEK1-Dependent Transcriptional Regulation of RAD54L Promotes Proliferation and Radio Resistance in Glioblastoma. Transl Oncol 2018, 11(1):140-146.

36. Li Z, Chen X, Xu D, Li S, Chan MTV, Wu WKK: Circular RNAs in nucleus pulposus cell function and intervertebral disc degeneration. Cell Prolif 2019, 52(6):e12704. 
37. Li H, He C, Wang X, Wang H, Nan G, Fang L: MicroRNA-183 affects the development of gastric cancer by regulating autophagy via MALAT1-miR-183-SIRT1 axis and PI3K/AKT/mTOR signals. Artif Cells Nanomed Biotechnol 2019, 47(1):3163-3171.

38. Tapia O, Riquelme I, Leal P, Sandoval A, Aedo S, Weber H, Letelier P, Bellolio E, Villaseca M, Garcia P et al: The PI3K/AKT/mTOR pathway is activated in gastric cancer with potential prognostic and predictive significance. Virchows Arch 2014, 465(1):25-33.

39. Cheng TL, Chen PK, Huang WK, Kuo CH, Cho CF, Wang KC, Shi GY, Wu HL, Lai CH:

Plasminogen/thrombomodulin signaling enhances VEGF expression to promote cutaneous wound healing. J Mol Med (Berl) 2018, 96(12):1333-1344.

40. Zhu L, Weng Z, Shen P, Zhou J, Zeng J, Weng F, Zhang X, Yang H: S100B regulates inflammatory response during osteoarthritis via fibroblast growth factor receptor 1 signaling. Mol Med Rep 2018, 18(6):4855-4864.

41. Wang Z, Wang J, Yang Y, Hao B, Wang R, Li Y, Wu Q: Loss of has-miR-337-3p expression is associated with lymph node metastasis of human gastric cancer. J Exp Clin Cancer Res 2013, 32:76.

42. Zhu S, Peng W, Li X, Weng J, Zhang X, Guo J, Huang D, Rong Q, Chen S: miR-1827 inhibits osteogenic differentiation by targeting IGF1 in MSMSCs. Sci Rep 2017, 7:46136.

43. Glentis S, Dimopoulos AC, Rouskas K, Ntritsos G, Evangelou E, Narod SA, Mes-Masson AM, Foulkes WD, Rivera B, Tonin PN et al: Exome Sequencing in BRCA1- and BRCA2-Negative Greek Families Identifies MDM1 and NBEAL1 as Candidate Risk Genes for Hereditary Breast Cancer. Front Genet $2019,10: 1005$.

44. Li J, Liu C, Li D, Wan M, Zhang H, Zheng X, Jie X, Zhang P, Li J, Hou H et al: OLFM4 Inhibits EpithelialMesenchymal Transition and Metastatic Potential of Cervical Cancer Cells. Oncol Res 2019, 27(7):763-771.

45. Bashamboo A, McElreavey K: NR5A1/SF-1 and development and function of the ovary. Ann Endocrinol (Paris) 2010, 71(3):177-182.

46. Zong D, Yin L, Zhong Q, Guo WJ, Xu JH, Jiang N, Lin ZR, Li MZ, Han P, Xu L et al: ZNF488 Enhances the Invasion and Tumorigenesis in Nasopharyngeal Carcinoma Via the Wnt Signaling Pathway Involving Epithelial Mesenchymal Transition. Cancer Res Treat 2016, 48(1):334-344.

47. Liu PF, Chen HC, Shu CW, Sie HC, Lee CH, Liou HH, Cheng JT, Tsai KW, Ger LP: Guanylate-binding protein 6 is a novel biomarker for tumorigenesis and prognosis in tongue squamous cell carcinoma. Clin Oral Investig 2020, 24(8):2673-2682.

48. Agange N, Sarraf D: Occult Macular Dystrophy with Mutations in the Rp1l1 and Kcnv2 Genes. Retin Cases Brief Rep 2017, 11 Suppl 1:S65-S67.

49. Seifert M, Przekopowitz M, Taudien S, Lollies A, Ronge V, Drees B, Lindemann M, Hillen U, Engler H, Singer BB et al: Functional capacities of human IgM memory B cells in early inflammatory responses and secondary germinal center reactions. Proceedings of the National Academy of Sciences of the United States of America 2015, 112(6):E546-555. 
50. Banerjee A, Shukla S, Pandey AD, Goswami S, Bandyopadhyay B, Ramachandran V, Das S, Malhotra A, Agarwal A, Adhikari S et al: RNA-Seq analysis of peripheral blood mononuclear cells reveals unique transcriptional signatures associated with disease progression in dengue patients. Transl Res 2017, 186:62-78 e69.

51. Risbud MV, Shapiro IM: Role of cytokines in intervertebral disc degeneration: pain and disc content. Nat Rev Rheumatol 2014, 10(1):44-56.

52. Zong D, Jiang N, Xu JH, Wang DJ, Zhu HF, Wu LR, Chen C, Yin L, He X: ZNF488 is an independent prognostic indicator in nasopharyngeal carcinoma and promotes cell adhesion and proliferation via collagen IV/FAK/AKT/Cyclin D1 pathway. Cancer Manag Res 2019, 11:5871-5882.

53. Fontes RBV, Baptista JS, Rabbani SR, Traynelis VC, Liberti EA: Normal aging in human lumbar discs: An ultrastructural comparison. PLoS One 2019, 14(6):e0218121.

54. Khan H, Reale M, Ullah H, Sureda A, Tejada S, Wang Y, Zhang ZJ, Xiao J: Anti-cancer effects of polyphenols via targeting p53 signaling pathway: updates and future directions. Biotechnol Adv 2020, 38:107385.

\section{Tables}

Table 1 Basic information of the 3 microarray datasets from GEO

\begin{tabular}{|llll|}
\hline Data source & Platform & Series & Sample size $(T / N)$ \\
\hline CircRNA & GPL19978 & GSE67566 & $5 / 5$ \\
\hline miRNA & GPL19449 & GSE63492 & $5 / 5$ \\
\hline mRNA & GPL21185 & GSE124272 & $8 / 8$ \\
\hline
\end{tabular}

\section{Figures}




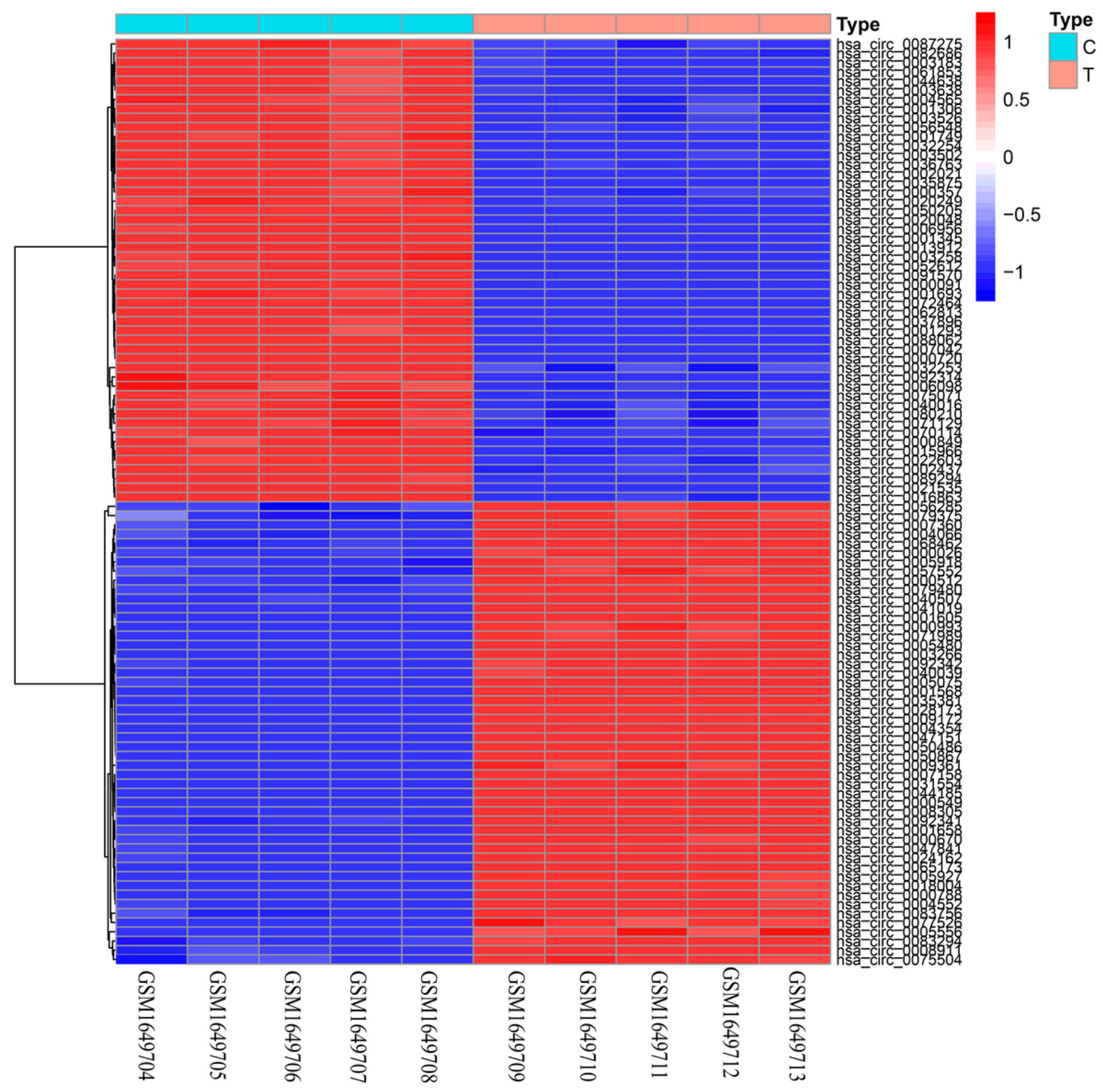

Figure 1

Heatmap of the 620 differentially expressed circRNAs in the 10 microarray datasets. By analysing the circRNA dataset, 620 differentially expressed circRNAs were identified ( $\mid \log 2$ (fold change (FC)) $\mid>1$ and an adjusted $P$ value $<0.05$ were considered $\mathbb{Z}$ 


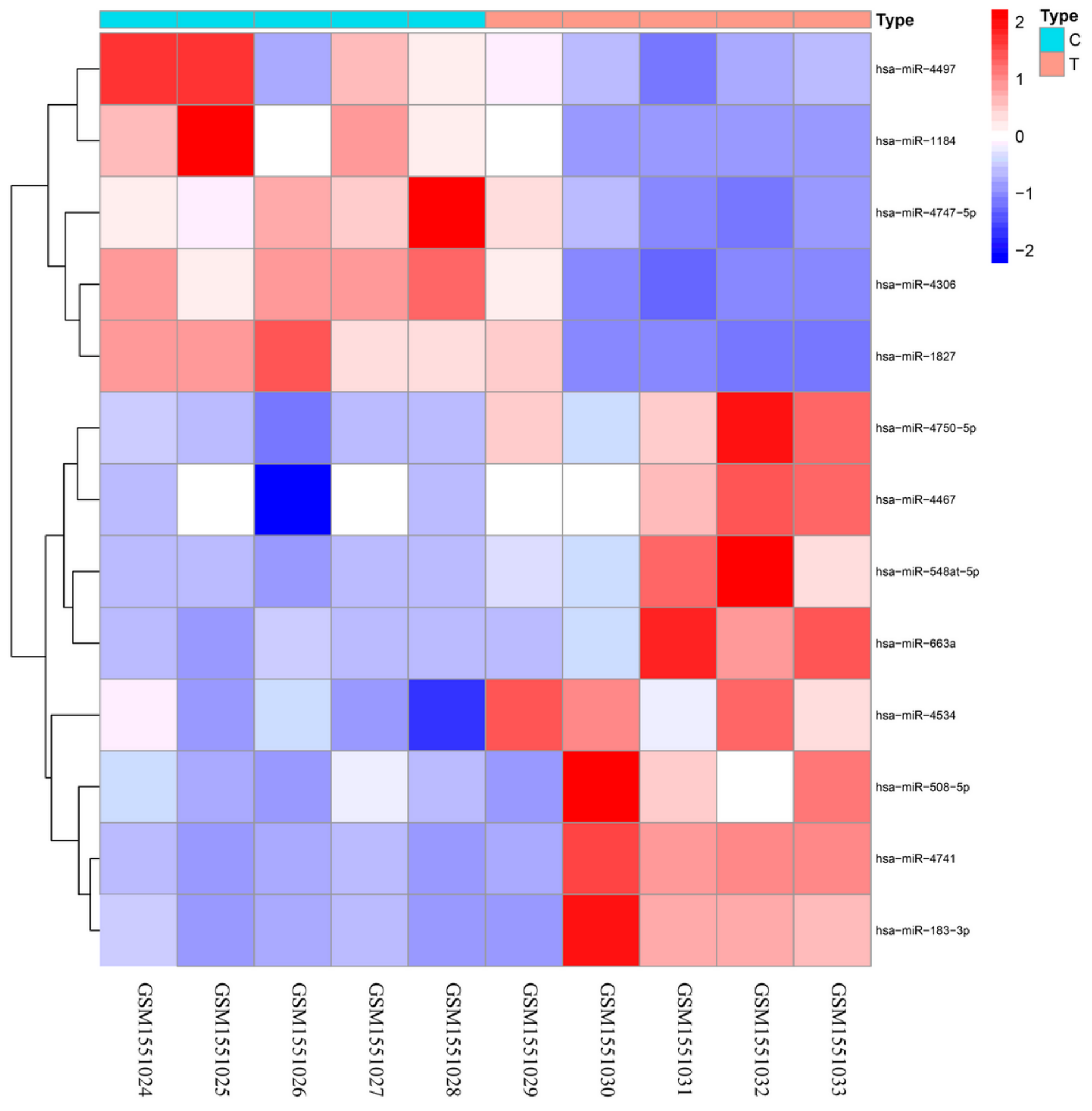

Figure 2

A total of 13 differentially expressed miRNAs were obtained by analysing the mRNA dataset (Ilog 2 (fold change $(\mathrm{FC})) \mid>1$ and an adjusted $\mathrm{P}$ value $<0.05$ were considered) 


\section{diff miRNA}

MRE

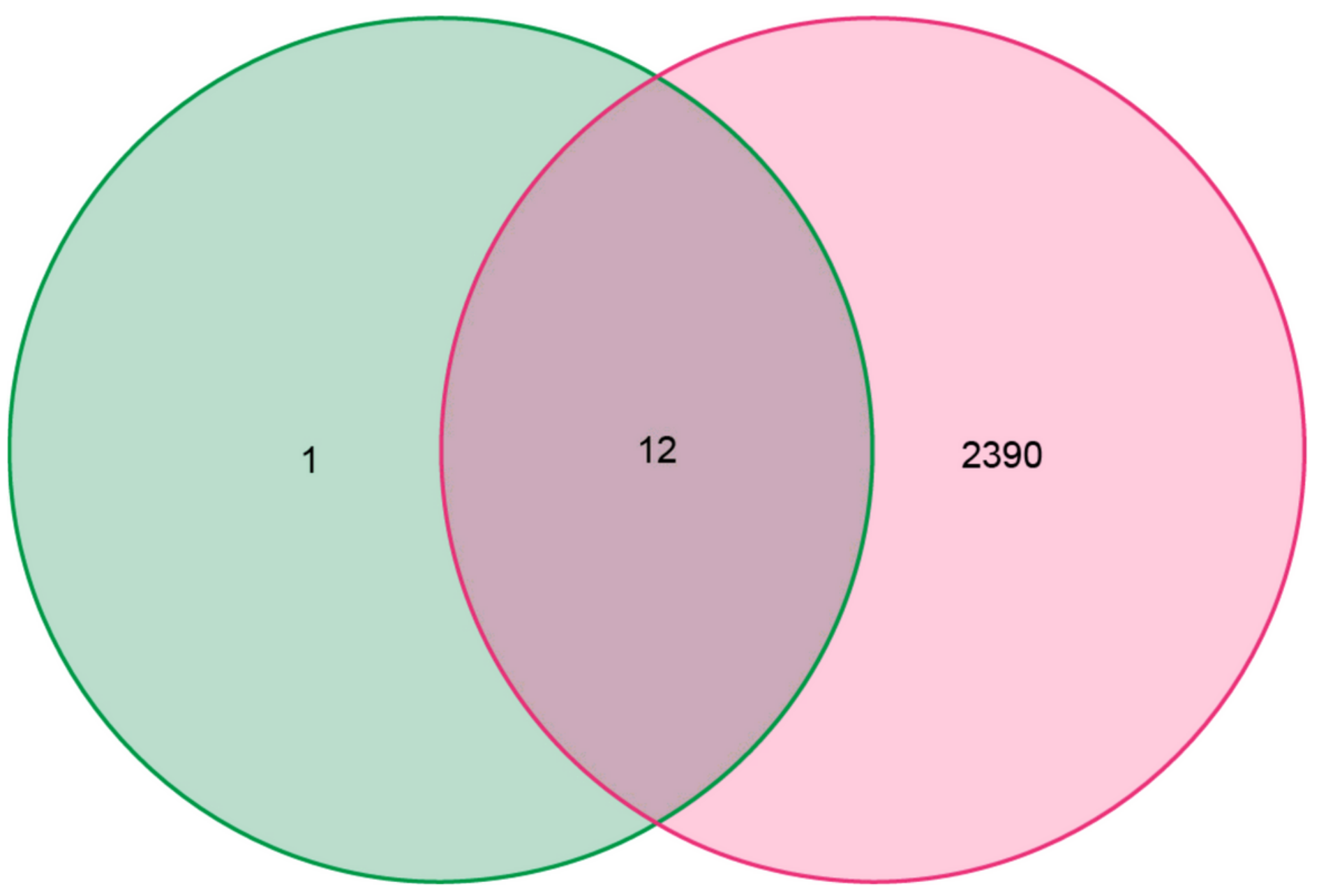

Figure 3

12 miRNAs were obtained from the intersection of miRNAs combined $\triangle M E R \rrbracket$ and the differentially expressed miRNAs 


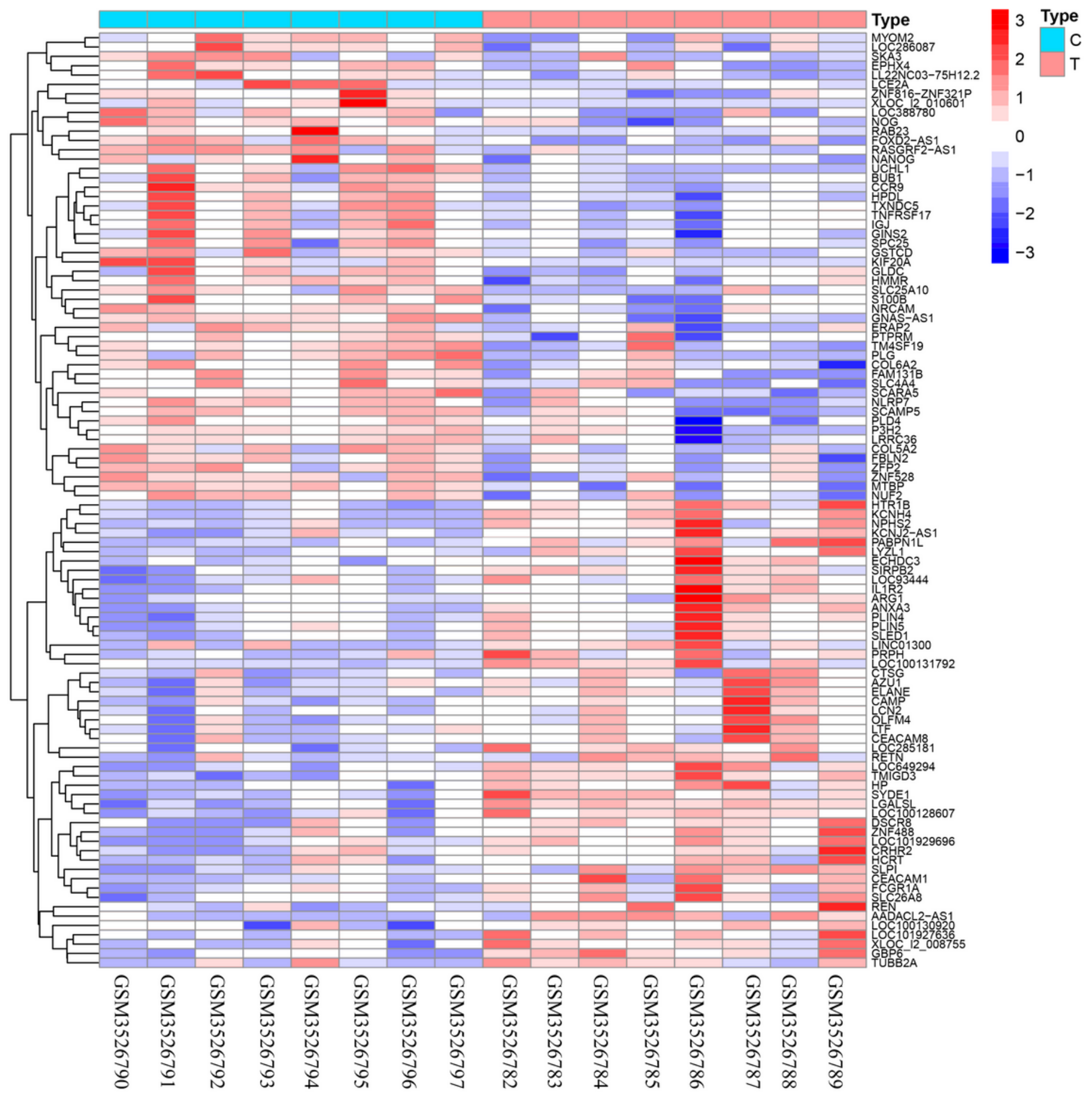

Figure 4

A total of 273 differentially expressed mRNAs were obtained by analysing the mRNA dataset (Ilog 2 (fold change $(\mathrm{FC})) \mid>1$ and an adjusted $\mathrm{P}$ value $<0.05$ were considered) 


\section{diff mRNA}

\section{miRNA target}

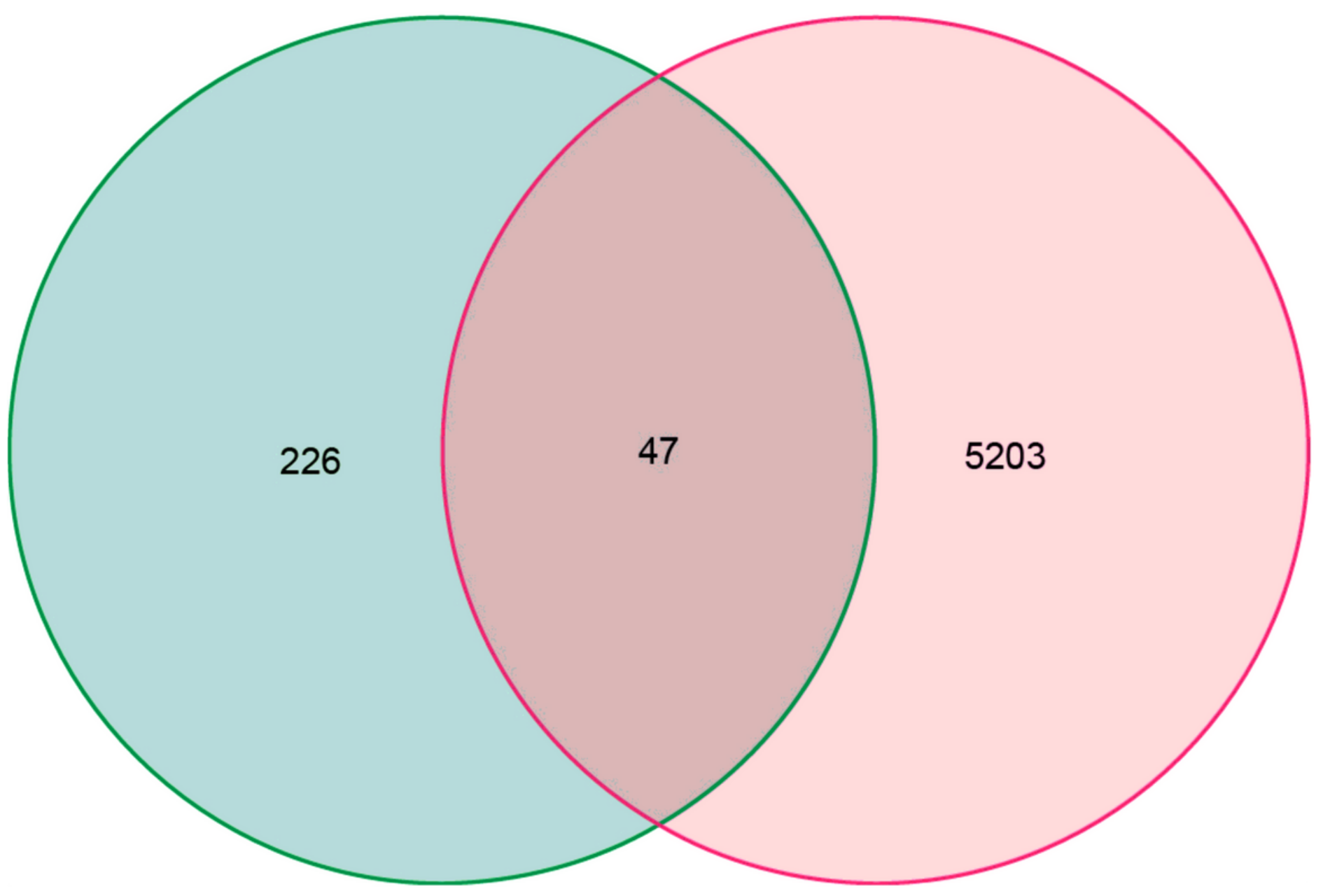

Figure 5

47 mRNAs were obtained from the intersection of mRNAs predicted and the differentially expressed mRNAs 


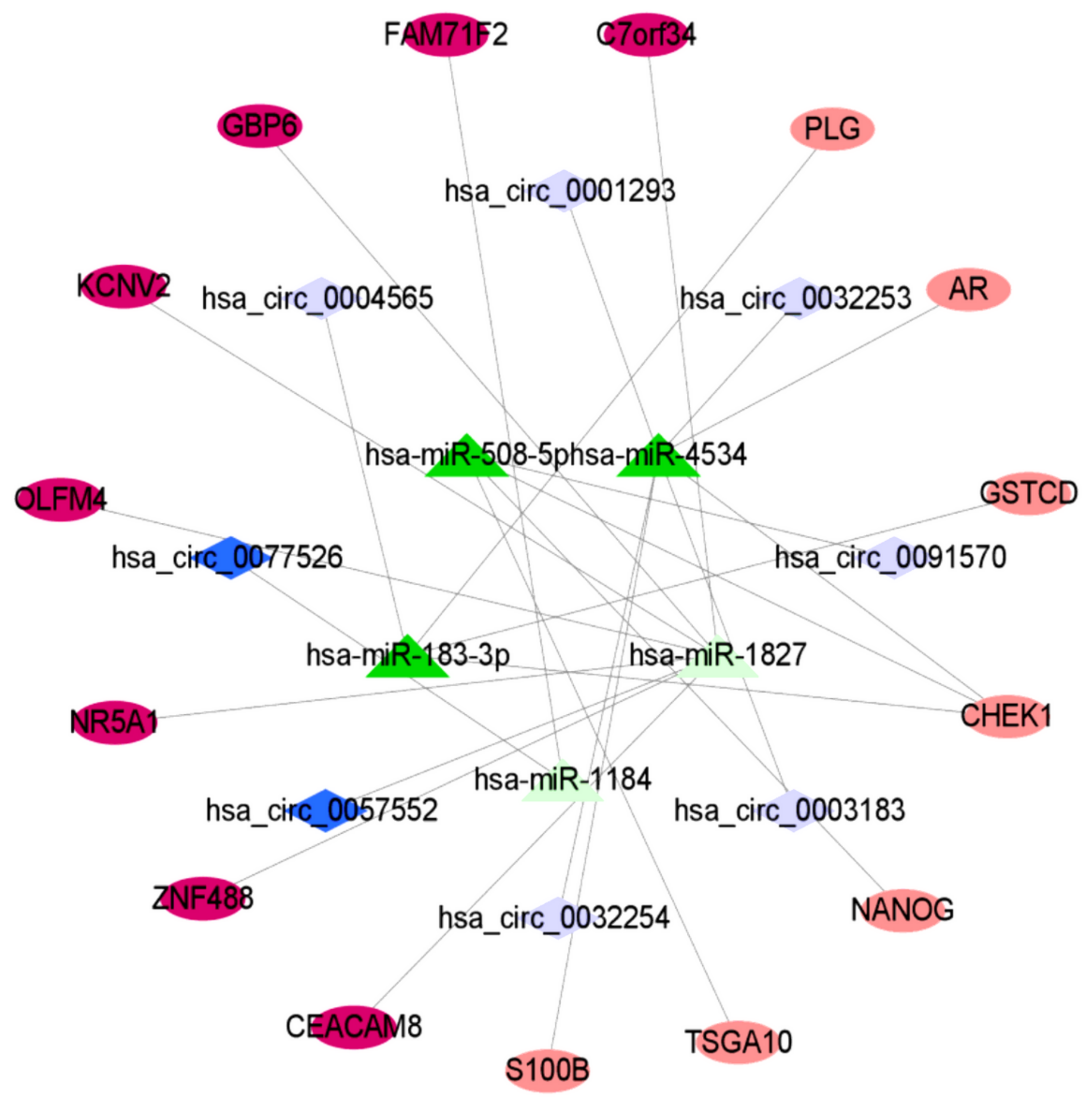

Figure 6

CeRNA network of circRNA-miRNA-mRNA interactions in IDD. Red indicates mRNAs, green indicates miRNAs, and blue indicates circRNAs. The deep color and light color represent up-regulation and down-regulation, respectively 

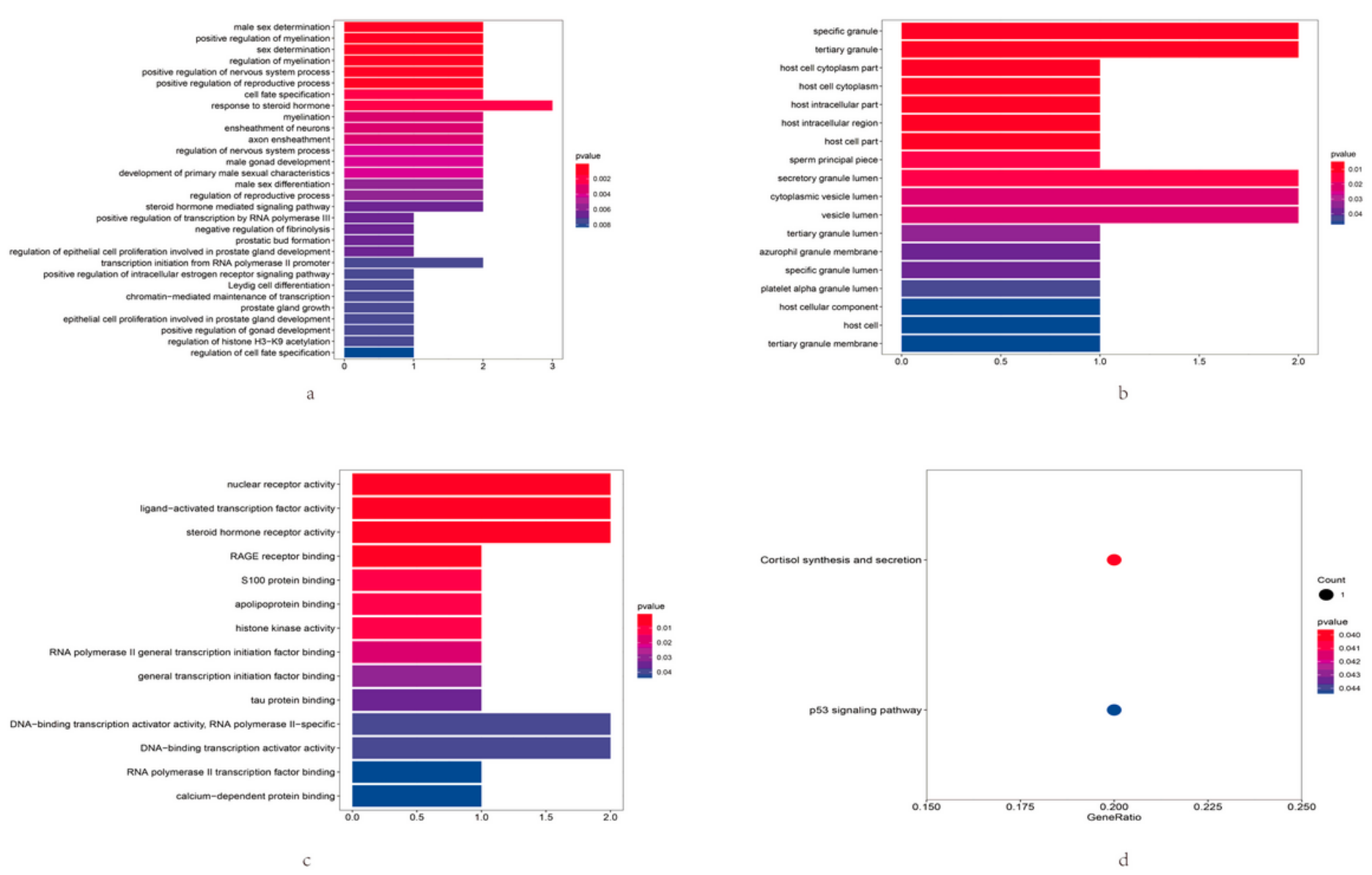

Figure 7

GO and KEGG functional enrichment analyses of the differentially expressed mRNAs in the ceRNA network. a: The enrichment of biological processes. b: The enrichment of cellular components. c: The enrichment of molecular functions. d: The enrichment analysis of the KEGG pathways. $P<0.05$ was considered to indicate a statistically significant difference 